\title{
Understanding Bohmian mechanics: A dialogue
}

\author{
Roderich Tumulka* \\ Dipartimento di Fisica and INFN sezione di Genova, \\ Università di Genova, Via Dodecaneso 33, 16146 Genova, Italy
}

This paper is an introduction to the ideas of Bohmian mechanics, an interpretation of quantum mechanics in which the observer plays no fundamental role. Bohmian mechanics describes, instead of probabilities of measurement results, objective microscopic events. In recent years, Bohmian mechanics has attracted increasing attention by researchers. The form of a dialogue allows me to address questions about the Bohmian view that often arise.

\section{FIRST DAY: FUNDAMENTALS}

Alice: What, exactly, does Bohmian mechanics say?

Bob: It describes the motion of $N$ point particles in the usual three-space. Every particle $i$ has at every time $t$ some definite position $Q_{i}(t) \in \mathbb{R}^{3}$. The motion obeys the first-order differential equation

$$
\frac{d Q_{i}}{d t}=\frac{\hbar}{m_{i}} \operatorname{Im} \frac{\nabla_{i} \Psi\left(Q_{1}(t), \ldots, Q_{N}(t), t\right)}{\Psi\left(Q_{1}(t), \ldots, Q_{N}(t), t\right)}
$$

where Im means the imaginary part, $m_{i}$ is the mass of particle $i$, and $\Psi$ is a timedependent complex-valued function on the configuration space $\mathbb{R}^{3 N}$ that satisfies Schrödinger's equation

$$
i \hbar \frac{\partial \Psi}{\partial t}=-\sum_{i=1}^{N} \frac{\hbar^{2}}{2 m_{i}} \nabla_{i} \cdot \nabla_{i} \Psi+V\left(q_{1}, \ldots, q_{N}\right) \Psi
$$

where $V$ is the potential energy. (We denote the variables on which $\Psi$ depends by $q$, and the actual positions of the particles by $Q$.)

Alice: And this mechanics is intended to replace nonrelativistic quantum mechanics?

Bob: Yes. The idea is that Bohmian mechanics is the true quantum mechanics. The $\Psi$ function is the very same wave function you know from quantum mechanics, and the 
positions of the particles are the same you would find if you performed a position measurement in quantum mechanics.

Alice: So the Bohmian answer to "wave or particle?" is "wave and particle!"

Bob: Yes.

Alice: But, it's very different from the usual quantum mechanics conceptually, isn't it? Indeed, it's not a quantum theory at all; it's a classical theory.

Bob: It is indeed very different from the usual quantum mechanics conceptually. Usually, it is assumed that quantum particles don't have trajectories. Bohmian mechanics has in common with classical theories that it tells us a clear story about what's happening. On the other hand, as we will soon see, Bohmian mechanics is in perfect agreement with all probabilistic predictions of quantum mechanics. So, you are mistaken thinking that Bohmian mechanics is not a quantum theory; remember that its empirical implications agree with quantum mechanics (whenever quantum mechanics is unambiguous), and disagree with Newtonian mechanics. A corollary of this agreement is that Bohmian mechanics is confirmed by experience. In particular, the mere existence of Bohmian mechanics proves that the usually assumed nonexistence of trajectories cannot be concluded from experiment.

Alice: You will have to explain the agreement with the predictions of quantum mechanics. But, first I have some questions on the dynamics. Apparently, you have to assume that the wave function is not merely square integrable, but is differentiable.

Bob: We do assume that the wave function is differentiable (except perhaps at a few exceptional configurations).

Alice: For all times?

Bob: For all times. For a reasonably large class of potentials (including Coulomb), there is a dense subspace in the $L^{2}$ Hilbert space of wave functions that will be differentiable for all times (with few exceptional configurations).

Alice: And the equation of motion is ill-defined for all nodes, that is, zeros, of the wave function. What if your trajectory $\left(Q_{1}(t), \ldots, Q_{N}(t)\right)$ runs into a node? 
Bob: It has been proved $\mathbb{1}^{1}$ that for almost all initial configurations (according to the appropriate measure) and for all wave functions from a suitable class, the equation of motion has a unique global solution (that is, for all $t$ ). Hence, with "probability one" Bohmian trajectories never run into the singularities of the velocity field, that is, the nodes and the points where the wave function is not differentiable.

Alice: What is this appropriate measure?

Bob: The natural measure for counting initial configurations (that is, for talking about the size of a set of initial configurations) for the equation of motion with wave function $\Psi(t=0)$ is

$$
\left|\Psi\left(q_{1}, \ldots, q_{N}, 0\right)\right|^{2} d^{3 N} q,
$$

where $d^{3 N} q$ is the volume measure on configuration space. The measure (3) defines a measure on the set of solution curves $\left(Q_{1}(t), \ldots, Q_{N}(t)\right)$ of the equation of motion.

Alice: Why don't we simply count initial conditions by the volume measure?

Bob: For every measure on configuration space, the dynamics will transport its density function $\rho\left(q_{1}, \ldots, q_{N}, t\right)$ according to the continuity equation

$$
\frac{\partial}{\partial t} \rho+\sum_{i} \nabla_{i} \cdot\left(\rho \frac{\hbar}{m_{i}} \operatorname{Im} \frac{\nabla_{i} \Psi}{\Psi}\right)=0 .
$$

If we start with the volume measure, that is, $\rho=1$, at some time, the measure will cease to be the volume measure at other times. So, when starting with the volume measure, you arbitrarily prefer some point in time. Not so with the measure in Eq. (3). The measure $|\Psi(0)|^{2} d^{3 N} q$ is transported by the dynamics to the measure $|\Psi(t)|^{2} d^{3 N} q$. This can be easily checked by deriving the continuity equation

$$
\frac{\partial}{\partial t}|\Psi|^{2}+\sum_{i} \nabla_{i} \cdot\left(|\Psi|^{2} \frac{\hbar}{m_{i}} \operatorname{Im} \frac{\nabla_{i} \Psi}{\Psi}\right)=0
$$

from the Schrödinger equation. Eq. (5) means that the Bohmian velocity $\left(\hbar / m_{i}\right) \operatorname{Im}\left(\nabla_{i} \Psi / \Psi\right)$ equals $j_{i} /|\Psi|^{2}$, where $j_{i}$ is the probability current density (for particle $i$ ) of the wave function.

Alice: So, what you're saying is that the only way (for generic $\Psi$ ) to define a measure on the set of solution curves $\left(Q_{1}(t), \ldots, Q_{N}(t)\right)$ without preferring some point in time is by Eq. (3). 
Bob: Precisely.

Alice: Quantum mechanics says that $|\Psi(q, t)|^{2}$ is the probability density of finding the particles at configuration $q$ when measuring the positions at time $t$. If position measurements simply reveal the Bohmian positions, the Bohmian positions must be random and distributed according to $|\Psi|^{2} d^{3 N} q$.

Bob: We have to keep in mind that the wave function we are talking about is the wave function of all particles in the universe. When we talk about the distribution of measured positions, what we are considering is an ensemble of small subsystems, all within the same universe, and all having the same subsystem wave function $\psi$. It has been shown ${ }^{2}$ that for the overwhelming majority [according to the measure (3)] of possible initial configurations of the Bohmian universe, the configurations of these subsystems look as if they are random and independently $|\psi|^{2}$-distributed. We may think of the initial configuration of our universe as being random, but such an assumption is not needed here (and perhaps wouldn't make much sense, just as we don't regard the dimension of space as a random number). For a subsystem with wave function $\psi$, we may always assume the configuration to be random and $|\psi|^{2}$-distributed. This statement is called the quantum equilibrium hypothesis. ${ }^{2}$

Alice: What about the collapse or reduction of the wave function? Equation (2) implies there is no collapse. But, in the standard version of quantum mechanics, the collapse rule is required for the theory to give the correct results. Doesn't Bohmian mechanics need the collapse as well?

Bob: No, Bohmian mechanics doesn't need an additional collapse postulate. To see why, we have to distinguish again between the wave function $\Psi$ of the universe and the wave function $\psi$ of a subsystem. Since the evolution of $\Psi$ is described by Equation (2) at all times, $\Psi$ never collapses, as you said. In contrast, the wave function $\psi$ of the part of the universe on which we do an experiment does effectively collapse as a consequence of Eqs. (11) and (2).

Alice: You mean, you can derive the collapse from Eqs. (11) and (2)? It is well known that the collapse is nonunitary and therefore is in conflict with the Schrödinger evolution! 
Bob: We can derive the collapse. You will see. For simplicity, we consider a "measurement" with only two possible outcomes. And, let us first suppose a special form of the wave function of the universe, $\Psi=\psi \otimes \phi \otimes \Phi$, where $\psi$ is the wave function of the subsystem on which we perform the "measurement," $\phi$ is the wave function of the measuring apparatus, and $\Phi$ is that of the rest of the world. The symbol $\otimes$ denotes the tensor product of functions, that is, $\Psi(x, y, z)=\psi(x) \phi(y) \Phi(z)$, where $x, y, z$ are the configurations of subsystem, apparatus, and the rest of the world, respectively. $\Phi$ will be irrelevant to our discussion, so we ignore it here.

Alice: $\Phi$ is irrelevant because, as long as $\Psi$ is a product such as (something) $\otimes \Phi$, Eq. (1) implies that the motion of the subsystem and apparatus particles is independent of what's happening outside.

Bob: Yes. Suppose $\hat{U}$ is the unitary operator that represents the time evolution of the wave function during the "measurement" process.

Alice: Wait a second: why do you always put these quotation marks around the word "measurement"?

Bob: Because we should not expect that anything is actually being measured during what is usually called a "measurement." I'll return to this point later.

Alice: Hm. Go on.

Bob: Suppose $\phi_{0}$ is the wave function of the apparatus before the measurement, $\phi_{1}$ is that corresponding to the result 1 , and $\phi_{2}$ is that corresponding to result 2 . If $\psi_{1}$ is the eigenfunction corresponding to result 1 and $\psi_{2}$ the eigenfunction corresponding to result 2, we must have that

$$
\begin{aligned}
& \hat{U}\left(\psi_{1} \otimes \phi_{0}\right)=\psi_{1} \otimes \phi_{1}, \\
& \hat{U}\left(\psi_{2} \otimes \phi_{0}\right)=\psi_{2} \otimes \phi_{2} .
\end{aligned}
$$

Now, if $\psi=c_{1} \psi_{1}+c_{2} \psi_{2}$ is not an eigenfunction of the self-adjoint operator (the "observable") corresponding to this "measurement," then the linearity of the Schrödinger equation implies that

$$
\hat{U}\left(\psi \otimes \phi_{0}\right)=c_{1} \psi_{1} \otimes \phi_{1}+c_{2} \psi_{2} \otimes \phi_{2} .
$$


The wave functions $\phi_{1}$ and $\phi_{2}$ will have very disjoint configurational support, that is, $\phi_{1}$ and $\phi_{2}$ are supported by the sets $S_{1}$ and $S_{2}$, respectively, in the configuration space of the apparatus particles, and these two sets will not only be disjoint, but very far apart in configuration space, as they are macroscopically distinct. (The wave function $\phi_{1}$ will not strictly be zero outside $S_{1}$, but will be very close to zero, such that, say, $99.9 \%$ of $\left|\phi_{1}\right|^{2}$ will be concentrated in $S_{1}$; similarly for $\phi_{2}$ and $S_{2}$.)

Alice: Then, if the result is displayed by the position of a pointer (with $10^{23}$ particles) on a scale, all configurations in $S_{i}$ will have the positions of all pointer particles close to $i$, and so the elements of $S_{1}$ and $S_{2}$ will differ by one length unit in at least $10^{23}$ variables.

Bob: Yes. For all practical purposes, it will be impossible to have any interference between the two wave packets on the right-hand side of Eq. (7), because for interference, the supports of the two packets have to overlap in configuration space.

Alice: I see.

Bob: So far we have discussed only the wave function. Now, in Bohmian mechanics, the configuration point of subsystem + apparatus will be, thanks to the quantum equilibrium hypothesis, random and distributed according to $\left|c_{1} \psi_{1} \otimes \phi_{1}+c_{2} \psi_{2} \otimes \phi_{2}\right|^{2}$, which for disjointness of supports equals $\left|c_{1}\right|^{2}\left|\psi_{1}\right|^{2}\left|\phi_{1}\right|^{2}+\left|c_{2}\right|^{2}\left|\psi_{2}\right|^{2}\left|\phi_{2}\right|^{2}$. Therefore, the configuration point will reside in the set $\{$ subsystem configurations $\} \times S_{1}$ with probability $\left|c_{1}\right|^{2}$, and in the set $\{$ subsystem configurations $\} \times S_{2}$ with probability $\left|c_{2}\right|^{2}$. Note that this result coincides with the probability predictions of quantum mechanics. Furthermore, if the configuration point resides in the first set, the output of the apparatus will (unambiguously) read 1.

Alice: And, in this case, where is the collapsed wave function of the subsystem after the measurement?

Bob: The future motion of the configuration point will depend only on the first wave packet $c_{1} \psi_{1} \otimes \phi_{1}$ because, as you can see in Eq. (11), the velocity depends only on the value of the wave function and its derivatives at the configuration point $\left(Q_{1}(t), \ldots, Q_{N}(t)\right)$, and the two wave packets never meet again. 
Alice: Aha. Furthermore, I recall that product wave functions such as $c_{1} \psi_{1} \otimes \phi_{1}$ lead to independent motion of subsystem and apparatus, and I can read off from Eq. (11) that $c_{1} \psi_{1}$ generates the same motion as $\psi_{1}$ since $c_{1}$ cancels in the quotient. Hence, the subsystem behaves as if it had wave function $\psi_{1}$.

Bob: Yes.

Alice: But somehow, I missed the point where the collapse comes about.

Bob: If $x, y, z$ are again the configuration of the subsystem, the apparatus and the rest of the world, respectively, and $X(t), Y(t), Z(t)$ is the solution of Eq. (11), we call $\psi_{\text {cond }}(x, t)=$ $\Psi(x, Y(t), Z(t), t)$ the conditional wave function of the subsystem. As long as there is no interaction between the subsystem and anything else, the conditional wave function obeys a Schrödinger equation, but ceases to do so during interaction. The conditional wave function collapses, but not so the wave function of the universe. And, in contrast to the orthodox collapse, the collapse of $\psi_{\text {cond }}$ takes place objectively, takes a finite amount of time, and does not depend on an observer's knowledge.

Alice: What happens to the second wave packet, $c_{2} \psi_{2} \otimes \phi_{2}$ ?

Bob: It leads an empty life. It evolves according to Schrödinger's equation, but it doesn't influence the configuration.

Alice: But if $\Psi$ never collapses, it isn't a product $\psi_{1} \otimes$ (something) after the experiment. And, we assumed it is a product in the beginning of our discussion of the measurement process. So, how do you treat any further measurement?

Bob: It isn't necessary to assume $\Psi$ is a product. We might have allowed a number of empty wave packets somewhere far away in configuration space. Suppose $\Psi_{\perp}$ is such a wave packet, so that $\Psi=\psi \otimes \phi \otimes \Phi+\Psi_{\perp}$ while the support of $\Psi_{\perp}$ is macroscopically disjoint from that of $\psi \otimes \phi \otimes \Phi$ (which contains the configuration point); then, our discussion still applies. In this case $\psi$ is called the effective wave function of the subsystem, $\underline{\underline{2}}$ and $\psi_{1}$ is the effective wave function of the subsystem after the "measurement."

Alice: If I understand you correctly, the outcome of the measurement in general depends on the microstate, that is, the configuration and the wave function, of the measurement 
apparatus. In particular, it depends on the details of $\phi$, and these details are subject to thermal fluctuations.

Bob: In principle, yes. But, for practically relevant experiments, it turns out that the configuration of the apparatus and the details of its wave function don't influence the outcome. The origin of the randomness is the unknown subsystem configuration. But, different experimental arrangements corresponding to the same self-adjoint operator may lead to different outcomes for the same $\psi$ and the same subsystem configuration.

Alice: So, the outcome can't be predicted given a self-adjoint operator and the state (configuration, wave function) of the subsystem?

Bob: In many cases, it can't. That's why "measurement" is quite a misnomer in this context, because it isn't at all a property of the subsystem that is being "measured."

Alice: According to Bohmian mechanics. But, in other interpretations...

Bob: At least you don't know in general. Ask yourself how you know that a different apparatus ("measuring" the same "observable") acting on the same subsystem wouldn't have given a different "measurement" result.

Alice: I'll have to think about this. In quantum mechanics "measurement" is never understood in the sense of simply revealing a preexisting quantity, but rather of forcing nature to choose a value.

Bob: All the more reason to regard the word "measurement" as a misnomer. The word suggests a meaning in the outcomes which in general the outcomes don't have. Nobody would call throwing a die a measurement, as the outcome is not a preexisting quantity.

Alice: What about the famous quantum paradoxes in Bohmian mechanics?

Bob: They get resolved (see, e.g., Ref. 3). Since Bohmian mechanics describes the motion of objectively existing particles, there can't be any paradoxes. 


\section{SECOND DAY: BOHMIAN VERSUS ORTHODOX QUANTUM MECHANICS}

Alice: I see that Bohmian mechanics is a possible explanation of the quantum world. But, the particle trajectories can't be observed!

Bob: The word "observe" is somewhat ambiguous. Strictly speaking, in a Bohmian universe, the particle paths actually can be observed. Let's consider, for example, a single particle, in a double-slit experiment. We finally observe the position of the arrival of every single particle on the screen and, because the equation of motion is of first order in time, we can calculate the entire trajectory from this position. For instance, we can decide whether the particle passed the left or the right slit, without disturbing the interference pattern: for symmetry reasons, all particles that passed the left slit hit the left half of the screen, while those that passed the right slit hit the right half of the screen.

Alice: But, your last proposition cannot be tested empirically.

Bob: It cannot be tested empirically. But, it's common for physical theories to have implications that cannot be tested empirically.

Alice: I didn't have in mind that you could "observe" the trajectory by calculating it.

Bob: Most observations, be it the mass of the sun or the charge of the electron, are not done directly, but involve calculations. I understand, of course, that you had in mind detecting the particle's position every tenth of a second. But, the interaction involved with this detection would influence the particle's future motion, so we won't see the trajectory the particle would have followed if its position hadn't been detected (though what we observe is a Bohmian trajectory as well). It's well known that detecting the particle at the slits of a double-slit experiment will make the interference fringes disappear.

Alice: Hence, the trajectory cannot be seriously observed, and the equation of motion cannot be tested directly.

Bob: Neither can the Schrödinger equation as we can't observe wave functions. 
Alice: Why can’t we observe wave functions?

Bob: Assume I prepare an atom with a certain wave function and I give it to you. You can't find out the wave function if I don't tell you.

Alice: I see. This fact follows indeed from the mathematical rules of the quantum formalism. But if you give me a million atoms with the same wave function, I can determine the wave function.

Bob: Yes, but I don't give you a million, I give you a single one.

Alice: But it's not clear if the wave function is something real. It may be rather the description of our knowledge about the particle.

Bob: Let's consider a gedanken experiment. Suppose a computer chooses a wave function randomly and prepares an atom with this wave function. Then, it prints out some data defining a pair of orthogonal subspaces of the Hilbert space, one of them containing the wave function it had chosen. And, then it prints out a note that says which of the two subspaces contained the chosen wave function, puts it into an envelope, and seals it. After that, the computer erases its knowledge about the wave function. Now, nobody knows the wave function of this atom, and nobody can possibly find out. But, nature still remembers the wave function of this atom, because we can, according to the rules of the quantum-mechanical formalism, carry out an experiment that has the two subspaces mentioned earlier as eigenspaces, break the seal, and compare the prediction with the actual result. (Strictly speaking, agreement between prediction and result doesn't imply the wave function was contained in one of the subspaces, but the whole procedure can be repeated, and the computer's prediction is always true.) According to the formalism, the machine can only accomplish certainty of its predictions if the wave function actually lies in the predicted subspace. So, the wave function of the atom is well defined (or "known to nature" or "real") even in those cases when nobody is aware of it.

Alice: Strictly speaking, you gave an example of one case in which the wave function is well defined although nobody knows it. This example doesn't imply it is always well defined. 
Bob: Strictly speaking, you're right about that. But, it suggests that wave functions are always well defined, and at least it shows that the wave function is not merely a mathematical expression of the observer's knowledge. And, it shows that there exist things we can't observe.

Alice: If I understand you properly, what you're emphasizing is we can't directly check Schrödinger's equation by means of (i) measuring the wave function (without disturbing it); (ii) letting it evolve an amount of time; (iii) measuring the wave function again; and (iv) comparing the result with a numerical extrapolation using Schrödinger's equation.

Bob: Yes. Isn't that true?

Alice: Certainly. And, you're saying I shouldn’t complain about invisible trajectories as long as I accept Schrödinger's equation.

Bob: You can put it that way. You can, of course, test both Eqs. (11) and (2) by their more indirect consequences.

Alice: But, how do I know the correct description of reality is Bohmian mechanics rather than any other interpretation of quantum mechanics?

Bob: There is hardly any other interpretation that is consistent, accepts the existence of an outside reality, and agrees with the predictions of the quantum formalism. (For discussions of other interpretations, see Ref. 4 and 5.) In fact, the formalism itself suggests Bohmian mechanics. Let me explain how. Recall that the formalism states that the wave function evolves according to Schrödinger's equation unless we perform a "formal measurement." Every formal measurement is characterized by a self-adjoint operator, the possible "measurement results" are the eigenvalues of this operator, the probability of a certain result is the norm squared ...

Alice: ... of the projection of the wave function to the corresponding eigenspace, and this projection is the new wave function that remains after the "formal measurement."

Bob: Note that there is an ambiguity in the formalism because it is not completely clear which processes are formal measurements. In particular, we might either guess the 
wave function of the measurement apparatus, use Schrödinger's equation for calculating the wave function of the composite system (object + apparatus) after the measurement, and then invoke the collapse rule when reading off the pointer position (or computer printout), or we might guess the self-adjoint operator corresponding to this apparatus and right away assume a collapse of the object wave function.

Alice: It is well known and easy to show that this ambiguity does not influence the set of possible results nor their probabilities or probabilities for future formal measurements, and hence the formalism is unambiguous.

Bob: In so far as macroscopic predictions are concerned. But, because we saw that the wave function (of the composite system) is well defined in reality, the question arises: when does the wave function collapse in reality? If you find it difficult to believe that the universe switches off the natural evolution law for a moment in favor of a different dynamics collapsing the wave function, then apparently the wave function never collapses. In this case, however, the wave function of the composite system will, in general, be a superposition of very different states, including different laboratory protocols or whatever [cf. Eq. (7)]. In particular, the result is not encoded in this wave function. Neither is there any randomness appearing.

Therefore, the wave function cannot be the complete description of the state of the composite system. There have to be additional variables that contain the actual result of the formal measurement. Such variables often are called "hidden variables" because they're not part (or functionals) of the wave function. But, this name turns out to be a misnomer if you remember that these variables contain the visible result, the only thing visible, in fact. Now, the question is, what are these additional variables? Let's see what the formalism suggests: the wave function is a function of the configuration, that is, of the particle positions. So, what's simpler than assuming that "particles" means particles and that a configuration actually exists? Indeed, what would be the meaning of the wave function being a function of the particle positions if there were no particle positions? If we assume that quantum particles have trajectories too, then the motion of these particles should be guided by the wave function. The precise formula of Eq. (11) can be obtained as the simplest one defining a Galilean invariant theory: ${ }^{2}$ 
Alice: I suppose that whoever says that the orthodox view of quantum mechanics is wrong should explain where mistakes were made on the way leading to this view.

Bob: The founders of quantum mechanics were much attracted by the thought that the words "momentum," "energy," and "angular momentum" still have a meaning in quantum mechanics. These words, however, don't have an immediate meaning (in contrast to "position," which does); their meaning in Newtonian mechanics comes from the fact that they are conserved quantities. Without this fact, nobody would be interested in multiplying mass by velocity. Now, Newtonian mechanics has turned out wrong, so naively we should expect that these words cease to have a meaning. But, Heisenberg and others insisted they have a meaning. The idea was that to define

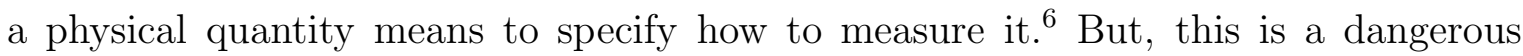
strategy because you don't know whether your result depends on the details of your measurement arrangement. There's no problem with defining a quantity by specifying how to measure it as long as you can predict the values. Then, you can be sure the value didn't depend on the arrangement. But, there is a problem as soon as the values are random. You don't even know you measured anything meaningful, because whatever definition-in-terms-of-how-to-measure you choose, it will always produce some result.

And, it is interesting which definitions Heisenberg chose: the definitions he gave were always such that in a Newtonian world, they would have measured the Newtonian value (of momentum, energy, or angular momentum, respectively). Isn't that strange? Shouldn't we suspect that the correct experimental arrangement for measuring momentum (if such a quantity exists) in a world whose rules differ from Newton's might differ from that in Newton's world? Insisting on the belief that Newtonian momentum (energy, angular momentum) measurements reveal the momentum (energy, angular momentum) leads to the orthodox view of quantum mechanics.

Alice: Is there an "actual momentum" in Bohmian mechanics like the "actual position?"

Bob: You might define $m \dot{Q}$ as the actual momentum (but it is not a conserved quantity), or you might define $\langle\psi|(-i \hbar) \nabla| \psi\rangle$ as the actual momentum (which is a conserved quantity as long as translation invariance is satisfied). But, I doubt that such a definition will 
be helpful for calculations or for anything, as these quantities need not agree with the outcome of a "momentum measurement."

Alice: There is a pretty symmetry in quantum mechanics between position and momentum. Bohmian mechanics destroys that symmetry.

Bob: There is no such symmetry in quantum mechanics. The Hamiltonian breaks it. The Schrödinger equation is a differential equation in the position representation of the wave function, but it is only a pseudodifferential equation in momentum representation and just some operator equation in representations using other bases of Hilbert space.

Alice: But, you can choose a basis in Hilbert space. That's the symmetry.

Bob: You may as well Fourier transform Maxwell fields. But, that doesn't mean there is a symmetry in classical electrodynamics between physical (position) space and Fourier space.

\section{THIRD DAY: SPECIAL ISSUES}

Alice: What about spin in Bohmian mechanics?

Bob: We can replace the Schrödinger equation by the Pauli equation and Eq. (11) by

$$
\frac{d Q_{i}}{d t}=\frac{\hbar}{m_{i}} \operatorname{Im} \frac{\sum_{s} \Psi_{s}^{*} \nabla_{i} \Psi_{s}}{\sum_{s} \Psi_{s}^{*} \Psi_{s}},
$$

where $s$ is the spin index. It is understood that all functions ( $\Psi_{s}$ and its derivatives) are evaluated at the actual configuration.

Alice: So, there is no "actual spin vector?"

Bob: No. The spin is rather a property of the wave function.

Alice: What about identical particles? The wave function has to be antisymmetric for fermions and symmetric for bosons.

Bob: OK, let the wave function be antisymmetric, respectively, symmetric.

Alice: Nothing special otherwise? The same equation of motion? 
Bob: Nothing special. The same equation of motion.

Alice: But, the particles are still labeled by the numbers $1, \ldots, N$ in Eq. (11), whereas identical particles should not have such a labeling.

Bob: For symmetric or antisymmetric wave functions, Eq. (11) is invariant under permutations of the labels, so the unphysical labeling does not affect the trajectories.

Alice: Something else: The ground state of the hydrogen atom is a real-valued wave function. So, the Bohmian electron does not move. It stands still.

Bob: Yes.

Alice: That's counterintuitive.

Bob: Counterintuitive doesn't mean much. It may seem counterintuitive that, according to Maxwell's theory, the energy in a power cord is not transported within the wires but within the insulator. For my part, I don't have too much intuition about the interior of a hydrogen atom. Perhaps you can explain your intuition to me.

Alice: Well, the nucleus exerts a Coulomb force on the electron, and in a stable atom this force should be compensated by some centrifugal force.

Bob: So, you mean (Coulomb force) $+($ centrifugal force $)=0$ ? Well, the centrifugal force is, in general, $-m \ddot{x}$, right? So, your argument implies $m \ddot{x}=$ (Coulomb force). This relation is precisely Newtonian mechanics, and we can experimentally test Newtonian against Bohmian mechanics. Bohmian mechanics wins.

Alice: But, from quantum mechanics one expects that if particle paths are to make sense, they should be Newtonian.

Bob: The existence of particle paths as such does not imply Newton's equation. It is a frequent prejudice that particle paths, if there are any, should be Newtonian paths. What you refer to in quantum mechanics is the fact that if a small wave packet stays a small wave packet for a time, its (only roughly defined) "path" is more or less Newtonian. But, this path is something different from the Bohmian particle path (which is always and precisely defined). 
Alice: OK, I'll give a different example. Suppose a particle is confined between two impermeable walls. Its wave function is a multiple of $e^{i k x}+e^{-i k x}$, where $k$ is chosen so that the wave function vanishes at the walls. Again, the Bohmian particle stands still.

Bob: Yes.

Alice: But, quantum mechanics says the momentum is, up to small corrections, either $\hbar k$ or $-\hbar k$, so the particle can't be at rest.

Bob: The word "momentum" doesn't have a meaning.

Alice: But, we can measure the momentum.

Bob: Tell me how you measure the momentum.

Alice: Take away the walls and let the particle move freely for an amount of time. Then, detect its position. If the amount of time was large enough and the distance between the walls small enough, we know quite precisely how far the particle traveled. Now, divide by time and multiply by mass.

Bob: The result of this experiment is perfectly predicted by Bohmian mechanics. The trajectory of the Bohmian particle in your experiment looks like this: it is a smooth curve $t \mapsto X(t)$ which is constant, $X(t)=x_{0}$, before the walls are removed and which is asymptotic to the line $X(t) \approx(\hbar k / m) t+$ constant if $x_{0}$ lies right of the center and asymptotic to the line $X(t) \approx-(\hbar k / m) t+$ constant if $x_{0}$ lies left of the center. Each of these two cases occurs with probability $1 / 2$.

Alice: So the particle slowly accelerates until it reaches the velocity $\pm \hbar k / m$ ?

Bob: Yes.

Alice: But, I always imagined the particle going back and forth between the walls, having velocity either $\hbar k / m$ or $-\hbar k / m$, each half of the time.

Bob: That's Newtonian mechanics, and Newtonian mechanics is refuted by experiment.

Alice: But, Newtonian mechanics for our experiment makes the true prediction that the particle will, with a certain fixed velocity, move either in the $x$ or in the $-x$ direction 
after the walls have been removed. So why should we give up Newtonian mechanics in this case?

Bob: Because it can't cope with other experiments, such as the double-slit.

Alice: I have another question. You said the wave function is something real. So, Bohmian mechanics says the wave function is something like a physical field.

Bob: If you wish to put it that way, yes.

Alice: But physical fields are always functions on three-space, not on configuration space. Probability densities are functions on configuration space.

Bob: The Maxwell and the gravity fields are functions on three-space, but this doesn't mean every physical field is a function on three-space. I can imagine having fields on configuration space. Why not? Indeed, I can simulate a Bohmian universe on a computer (by the way, it is very unclear how to simulate an orthodox quantummechanical universe on a computer); now, what should, say, intelligent life forms inhabiting this universe think about physical fields? These beings would be wrong about their world unless they regard $\Psi$ as a physical field on configuration space, as that is how I simulate it.

Alice: Isn't existence of actual particle positions more of a metaphysical question than a physical one?

Bob: An ancient astronomer might have said that the positions of the planets in threespace cannot be observed, and so we should restrict our theories to describing the motion of the planets on the two-sphere, against the background of fixed stars. Such a view would certainly have influenced physics, so it would not have been merely of metaphysical interest. That's why I can't see why the existence of trajectories should not be a physical question.

Alice: But, as there is no way of testing Bohmian mechanics against orthodox quantum mechanics experimentally, how do I know the trajectories exist?

Bob: "Is it not clear from the smallness of the scintillation on the screen that we have to do with a particle?" (J. S. Bell,,$^{7}$ p. 191). 
Alice: How do you know you have the correct trajectories? How do you know it won't turn out to be necessary to change the equation of motion one day?

Bob: In fact, I don't. But, that's not a tragedy. How do you know Schrödinger's equation is correct?

Alice: It certainly isn't. It's nonrelativistic.

Both: But, that's not a tragedy.

Alice: Why should a physicist deal with philosophical questions?

Bob: Bohmian mechanics is a differential equation. Not philosophy. It's the orthodox view that introduces a number of cryptic philosophical pronouncements for explaining away the problems of quantum mechanics.

Alice: There's one big objection against Bohmian mechanics: the majority of physicists believes in the quantum orthodoxy.

Bob: A philosopher, engineer, mathematician, or chemist might accept the authority of the majority of physicists. But, if you are a physicist yourself, you are in the position to decide for yourself.

Alice: A final question: How should we scientifically answer metaphysical questions?

Bob: The debate on Bohmian mechanics rather resembles the debate at the beginning of the 20th century on the question "In mechanical terms, what does entropy precisely mean and what does the second law of thermodynamics precisely state?" 8 than a metaphysical debate. I have to explain this comparison. Look, every physical theory we know is more or less ill-defined. Newton's $1 / r^{2}$ force law is ill-defined as soon as two particles collide, the Lorentz force evaluates the Maxwell field at a singular point, and there are dozens of other problems. Some of these problems we may safely ignore, some not. Some theories are better defined than others. My message is that the usual quantum mechanics is ill-defined in such a way that you should be dissatisfied with it. Now, the question is how to make sense out of the formalism of quantum mechanics. The meaning of entropy was discussed in statistical mechanics a hundred years ago, and it is the meaning of quantum mechanics that we are discussing now. 
And, Bohmian mechanics is the best way to make sense out of quantum mechanics. If you're wondering what does really happen during quantum processes, Bohmian mechanics is the most natural answer.

\section{FURTHER READING}

J. S. Bell's collected papers on the foundations of quantum mechanics ${ }^{7}$ contain many excellent articles on the essential problem with ordinary, orthodox quantum mechanics, and the existing possibilities for solving this problem. Bell calls Bohmian mechanics the "de Broglie-Bohm theory." Reference 3 is a nice short paper explaining how Bohmian mechanics solves a paradox.

Bohm's original papers are of historical interest. 9 You should keep in mind, however, that they represent the 1952 state-of-affairs, containing errors about the behavior of the solutions of Eq. (II) and speculations that have not led anywhere. Later in his life, Bohm wrote a book on Bohmian mechanics $\stackrel{4}{\underline{4}}$ together with B. J. Hiley. In this book, you will find pictures of Bohmian paths and detailed discussions of special topics. Another source of historical interest is the Fifth Solvay Congress of 1927,,$\frac{10}{n}$ where similar ideas were proposed by L. de Broglie. The history of Bohmian mechanics and its reception is outlined in Ref. 11.

A detailed overview of Bohmian mechanics can be found in Ref. 12. An overview of the mathematical research on Bohmian mechanics up to 1995 is given in Ref. 13. A comparison of Bohmian mechanics with other attempts at finding out what quantum mechanics means is made in Ref. 5 .

Reference 2, a long research paper, contains a detailed analysis of how to justify the quantum equilibrium hypothesis, and Ref. 14 discusses various aspects of quantum measurements from a Bohmian perspective. For extensions of Bohmian mechanics to quantum field theory, see Ref. 15 and the references therein, and for a perspective on a relativistic version of Bohmian mechanics, see Chap. 12 of Ref. 4 .

\section{Acknowledgments}

I owe my material, and many nice formulations, to the papers of John S. Bell, David Bohm, Detlef Dürr, Sheldon Goldstein, and Nino Zanghì. I am particularly grateful to 
Detlef Dürr for many discussions I had with him before writing this dialogue. I also thank Sheldon Goldstein and Travis Norsen for their helpful comments and suggestions.

* Electronic address: tumulka@mathematik.uni-muenchen.de

1 K. Berndl, D. Dürr, S. Goldstein, G. Peruzzi, and N. Zanghì, "On the global existence of Bohmian mechanics," Commun. Math. Phys. 173, 647-673 (1995).

2 D. Dürr, S. Goldstein, and N. Zanghì, "Quantum equilibrium and the origin of absolute uncertainty,” J. Stat. Phys. 67, 843-907 (1992). arXiv.org/quant-ph/0308039.

3 J. S. Bell, "De Broglie-Bohm, delayed-choice double-slit experiments, and density matrix," Int. J. Quantum Chem. 14, 155-159 (1980). Reprinted in Ref. 7, p. 111.

4 D. Bohm and B. J. Hiley, The Undivided Universe: An Ontological Interpretation of Quantum Theory (Routledge, London, 1993).

5 S. Goldstein, "Quantum theory without observers: Part one," Phys. Today 51(3), 42-46 (1998);

S. Goldstein, "Quantum theory without observers: Part two," Phys. Today 51(4), 38-42 (1998).

6 W. Heisenberg, "Über den anschaulichen Inhalt der quantentheoretischen Kinematik und Mechanik," Z. Phys. 43, 172-198 (1927). English translation p. 62-87 in Quantum Theory and Measurement, edited by J. A. Wheeler and W. H. Zurek (Princeton U. P., Princeton, 1983)

7 J. S. Bell, Speakable and Unspeakable in Quantum Mechanics (Cambridge U. P., Cambridge, 1987).

8 P. Ehrenfest and T. Ehrenfest, "Begriffliche Grundlagen der statistischen Auffassung in der Mechanik," (1911) in Enzyklopädie der Mathematischen Wissenschaften, Vol. IV-4, Art. 32 (Teubner, Leipzig, 1917).

9 D. Bohm, "A suggested interpretation of the quantum theory in terms of "hidden" variables. I," Phys. Rev. 85, 166-179 (1952); D. Bohm, "A suggested interpretation of the quantum theory in terms of "hidden" variables. II," Phys. Rev. 85, 180-193 (1952).

10 Electrons and Photons: The Proceedings of the Fifth Solvay Congress (1927), translated and edited by G. Bacciagaluppi and A. Valentini (Cambridge University Press, in preparation).

11 J. T. Cushing, Quantum Mechanics: Historical Contingency and the Copenhagen Hegemony (University of Chicago Press, Chicago, 1994).

12 S. Goldstein, "Bohmian mechanics," in Stanford Encyclopedia of Philos- 
ophy, edited by E. N. Zalta, published online by Stanford University $\langle$ http://plato.stanford.edu/archives/win2002/entries/qm-bohm/>.

13 K. Berndl, M. Daumer, D. Dürr, S. Goldstein, and N. Zanghì, "A survey of Bohmian mechanics," Nuovo Cimento Soc. Ital. Fis. 110B, 737-750 (1995).

14 D. Dürr, S. Goldstein, and N. Zanghì, "Quantum equilibrium and the role of operators as observables in quantum theory," J. Stat. Phys. 116, 959-1055 (2004). arXiv.org/quant-ph/0308038.

15 D. Dürr, S. Goldstein, R. Tumulka, and N. Zanghì, "Bohmian mechanics and quantum field theory," Phys. Rev. Lett. (in press). arXiv.org/quant-ph/0303156. 